10) Ohya, H., M, Okada, K. Okuno and Y. Negishi: Maku, 9, 285 (1984).

11) Ohya, H., M. Niwa, K. Matsumoto, T. Osada, Y. Baba and A. Komura: Maku, 10, 121 (1985).

12) Ohya, H., M. Niwa, M. Okada, K. Okuno, Y. Negishi, K. Matsumoto: Maku, 11, 115 (1986).

13) Ohya, H., M. Okada, K. Okuno and Y. Negishi: Maku, 10, 371 (1985).

14) Ohya, H., M. Okada, K. Okuno, Y. Negishi and M. Niwa: Maku, 10, 313 (1985).

15) Ohya, H., E. Kuwahara, Y. Ishizu, M. Niwa, Y. Negishi and K. Matsumoto: Maku, 10, 187 (1985).

16) Ohya, H., J. Taga, M. Okada, H. Yaguchi, T. Osada, Y. Baba and A. Komura: Maku, 8, 171 (1983)

17) Osada, T., Y. Baba, S. Komura, Y. Ishizu and H. Ohya: Maku, 9, 55 (1984).

18) Osada, T., Y. Baba, S. Komura, Y. Ishizu and H. Ohya: Maku, 9, 285 (1984).

19) Othmer, O. F. and R. F. Benenati: Ind. Eng. Chem., 37, 299 (1945).

20) Rozelle, L. T., J. E. Cadotte and K. E.: Cabian "Reverse Osmosis Synth. Membrn.," p. 249 (1977).

21) Spiegler, K. S. and O. Kedem: Desalination, 1, 311 (1966)

22) Staverman, A. J.: Rec. Trav. Chim., 70, 344 (1951).

23) Wilke, C. R. and P. Chang: AIChE J., 1, 264 (1955).

\title{
BUBBLE SIZE AND FREQUENCY IN GAS FLUIDIZED BEDS
}

\author{
JEONG H. CHOI AND JAE E. SON \\ Korea Institute of Energy and Resources, Dae Jeon, Korea \\ SANG D. KIM \\ Department of Chemical Engineering, Korea Advanced Institute of Science and \\ Technology, Seoul 131, Korea
}

Key Words: Bubble Size, Bubble Frequency, Bubble Velocity, Fluidized Bed

\begin{abstract}
A theoretical model for mean bubble size and frequency based on the collision theory with random spatial bubble distribution in freely bubbling gas-fluidized beds was developed. A hemispherical bubble velocity diagram was constructed to determine the average bubble collision frequency. The resulting theoretical equation for predicting bubble size is

$$
\left(U-U_{m f}\right)\left(D_{b}-D_{b 0}\right)+0.474 g^{1 / 2}\left(D_{b}^{3 / 2}-D_{b 0}^{3 / 2}\right)=1.132\left(U-U_{m f}\right) h
$$

Bubble characteristics such as pierced bubble length, velocity and point frequency were measured in a square $(30 \times 30-\mathrm{cm})$ fluidized bed by means of an electroresistivity probe.

The gradient of bubble size increased linearly with bubble voidage. The bubble Froude number also increased along the bed height with bubble voidage. The bubble Froude number represents approximately a linear relationship with the average fractional change of square root of the static energy of bubble rise along the bed height. The present model of bubble size was found to represent well the data in the literature.
\end{abstract}

\section{Introduction}

In freely bubbling fluidized beds, the hydrodynamic behaviour is mainly governed by bubble properties such as bubble velocity, voidage, and frequency. This information is essential in the analysis of fluidized bed behaviour. Also, it may indicate whether a fluidized bed is operated in the slugging or bubbling state.

The bubble properties in three-dimensional beds have been measured by means of electrical probes, by photographing the bubbles which are erupting from

\footnotetext{
Received June 22, 1987. Correspondence concerning this article should be addressed to S. D. Kim.
}

the bed surface, and by an X-ray photographic method.

A semiempirical correlation for predicting the frontal diameter of a bubble has been presented by Rowe, ${ }^{97}$ using a correlation of the number-density of bubbles determined by Rowe and Goldsmith. ${ }^{12)}$ Based on the literature data on bubble diameter, Darton et $a l .{ }^{3)}$ have proposed a semiempirical correlation for predicting the equivalent spherical bubble diameter.

It has been reported that bubbles tend to rise along certain preferred paths and that coalescence may take place with a considerable lateral movement of bubbles which may be slow enough to determine the overall 
growth rate of bubbles. ${ }^{3)}$

In the present study a theoretical model of bubble coalescence is proposed, based on the modified collision theory with random spatial bubble distribution. It is assumed that bubbles are moving randomly at equal speed along the preferred paths. Coalescence of bubbles can occur as a result of two bubbles colliding with each other in the bed. The existing correlations of bubble size are discussed in the context of the present model.

\section{Theory}

Bubbles may grow in size due to coalescence with other bubbles while they rise randomly from the distributor plate to the surface of the bed.

To describe such behaviour, it is assumed that 1) the bed is in freely bubbling state, 2) the bubble shape is spherical, 3) the bubble is rising uniformly across the bed without wall effect, 4) the two-phase theory holds, 5) coalescence is the only means of bubble growth, and 6) coalescence occurs between pairs of colliding bubbles. In addition, bubble breakage is not considered as in the cases of Geldart group B and D particles in the present model.

The number balance of bubbles over a bed layer of a thickness $\Delta h$ is shown in Fig. 1(a). The inlet flow rate across the surface at the height $h-\Delta h / 2$ is $\left.N_{f}\right|_{h-\Delta h / 2}$ and the outlet flow rate across the surface at the height $h+\Delta h / 2$ is $\left.N_{f}\right|_{h+\Delta h / 2}$.

The flow difference between the inlet and outlet must be equal to the number of coalescences or collisions in the given volume per unit time:

$$
\left.N_{f}\right|_{h-\Delta h / 2}-\left.N\right|_{h+\Delta h / 2}=
$$

$\{$ No. of coalescences in the given volume $\} / \mathrm{s}$

According to the collision theory, ${ }^{8)}$ accounting for the motion of all the bubbles, the collision frequency can be expressed as $\pi D_{b}^{2} V_{r} n$ in which $V_{r}$ is an average value of the relative speed of a pair of colliding bubbles.

Bubbles may move toward a preferred path, and their ascents are not always in the vertical direction but may be at an arbitrary angle relative to the vertical direction. For gas-fluidized beds, information on vertical bubble velocity and its point frequency is available. However, information on the angular velocity profiles and frequency are relatively sparse.

The most important bubble growth region is just above the distributor plate. In this region, random bubble movement can be resonably assumed. Then the time-averaged instantaneous bubble movement at a point of the distributor can be considered to be regular in angular frequency above the cross section. Therefore, an artificial bubble-velocity diagram is made in order to determine the average relative speed,
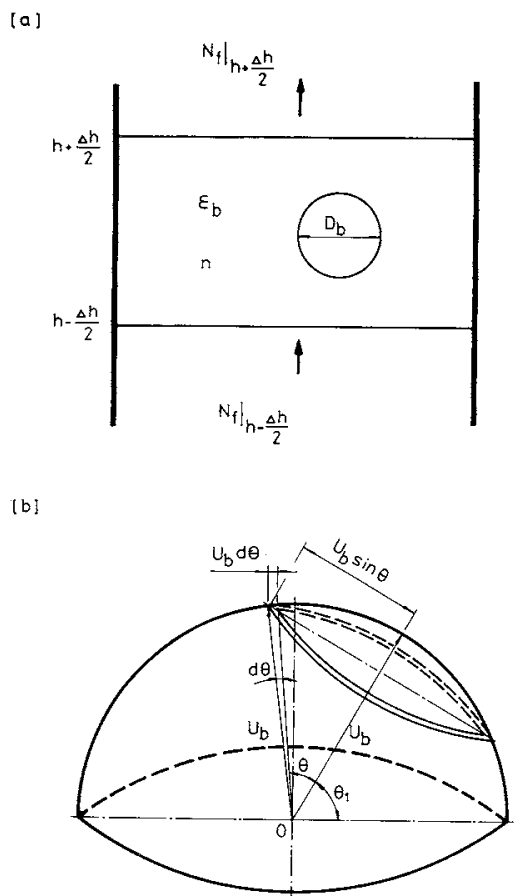

Fig. 1. (a): Number flow rate of bubble; (b): Mean angular bubble velocity diagram

$\bar{V}_{r}$, as shown in Fig. 1(b). In the figure, it is assumed that all the bubbles have the same speed, $U_{b}$, with a regular angular frequency.

When we consider a reference velocity vector having an angle, $\theta_{1}$, against the base plane and let $\theta$ be the angle between the reference vector and one of the vectors surrounding it, the relative velocity is the difference of the two velocity vectors. This is given by the thrid side of the triangle formed from the velocity vectors laid out from a common point. Since the triangle is isosceles, the relative speed, $V_{r}$, can be readily expressed as

$$
V_{r}=2 U_{b} \sin (\theta / 2)
$$

To determine the average relative speed in all the directions, it is necessary to know the probability of occurrence of an angle between $\theta$ and $\theta+d \theta$, which can be denoted as $P(\theta) d \theta$. If the angle between the two vectors lies between $\theta$ and $\theta+d \theta$, the terminus of the second vector will be situated on a thin band between the latitude circles, which is defined by the angle $\theta$ and $\theta+d \theta$. The probability of occurrence of an angle between $\theta$ and $\theta+d \theta$ is the ratio of the area of the thin band to the total hemisphere. Then the average relative speed, $\widetilde{V}_{r}$, over the total surface can be represented as

$$
\bar{V}_{r}=2 U_{b} \overline{\sin (\theta / 2)}
$$

in which $\overline{\sin (\theta / 2)}$ is $4 / 3 \sin ^{3}\left(\theta_{1} / 2\right)$ and $\theta$ lines within $0<\theta<\theta_{1}$.

Since the thin band is not a complete circle for 
$\theta_{1}<\theta<\pi-\theta_{1}$, the term $\overline{\sin (\theta / 2)}$ is expressed as

$$
\begin{aligned}
\overline{\sin (\theta / 2)} & =\int_{\theta_{1}}^{\pi / 2}\left\{\left[1-\frac{1}{\pi} \cos ^{-1}\left(\tan \theta_{1} / \tan \theta\right)\right]\right. \\
& \times \sin (\theta / 2) \sin \theta+\frac{\sqrt{2}}{2 \pi}\left[\cos \frac{\left(\theta-\theta_{1}\right)}{2}\right. \\
& \left.+\sin \frac{\left(\theta-\theta_{1}\right)}{2}\right] \cos \left(\theta-\theta_{1}\right) \cos ^{-1} \\
& \left.\times\left[\tan \left(\theta-\theta_{1}\right) \tan \theta_{1}\right]\right\} d \theta
\end{aligned}
$$

Hence, the collision frequency of a bubble moving with an angle, $\theta_{1}$, above the plane is given by

$$
F_{c s}=\pi D_{b}^{2} \bar{V}_{r} n=2 \pi D_{b}^{2} U_{b} n \overline{\sin (\theta / 2)}
$$

where

$$
\begin{aligned}
\overline{\sin (\theta / 2)}= & \frac{4}{3} \sin ^{3}\left(\theta_{1} / 2\right) \\
& +\int_{\theta_{1}}^{\pi / 2}\left\{\left[1-\frac{1}{\pi} \cos ^{-1}\left(\tan \theta_{1} / \tan \theta\right)\right]\right. \\
& \times \sin (\theta / 2) \sin \theta+\frac{\sqrt{2}}{2 \pi}\left[\cos \left(\frac{\theta-\theta_{1}}{2}\right)\right. \\
& \left.+\sin \left(\frac{\theta-\theta_{1}}{2}\right)\right] \cos \left(\theta-\theta_{1}\right) \cos ^{-1} \\
& \left.\left.\times\left[\tan \left(\theta-\theta_{1}\right) \tan \theta_{1}\right)\right]\right\} d \theta
\end{aligned}
$$

To calculate the number of bubbles per unit volume undergoing collision per unit time, Eq. (5) should be multiplied by the local probability for $\theta_{1}$ and bubble density and then integrated over the hemisphere for $\theta_{1}$.

$$
\begin{aligned}
N_{c v} & =\int_{0}^{\pi / 2} 2 \pi D_{b}^{2} U_{b} n \overline{\sin (\theta / 2)} n \cos \theta_{1} d \theta_{1} \\
& =2 \pi D_{b}^{2} U_{b} n^{2} \int_{0}^{\pi / 2} \overline{\sin (\theta / 2)} \cos \theta_{1} d \theta_{1}
\end{aligned}
$$

in which the probability of an arbitrary bubble in an angle between $\theta_{1}$ and $\theta_{1}+d \theta_{1}$ is $\cos \theta_{1} d \theta_{1}$. The integral in Eq. (7) can be evaluated numerically as 0.5658 .

The number of collisions, $F_{c v}$, per unit time per unit volume is given by one-half of $N_{c v}$ since the number of collisions is one-half the number of identical bubbles colliding.

The bubble density, $n$, can be expressed as $6 \varepsilon_{b} /$ $\left(\pi D_{b}^{3}\right)$. Therefore, the bubble balance of Eq. (1) is given by the limiting case of $\Delta h$ approaching zero:

$$
d N_{f} / d h=-\frac{20.37}{\pi} \frac{A U_{b} \varepsilon_{b}^{2}}{D_{b}^{4}}
$$

subject to the initial condition, $N_{f}=N_{f 0}$. From the two-phase theory, the number-based bubble flow rate, $N_{f}$, can be expressed as $6 k\left(U-U_{m f}\right) A / \pi D_{b}^{3}$.

The number-based bubble flow rate at the distributor plate, $N_{f 0}$, can be determined by substituting the initial mean bubble diameter at the disributor, $D_{b 0}$ for $D_{b}$.

The initial bubble diameter can be calculated by using the correlation of Darton et al. ${ }^{3 \text { ) }}$

The bubble voidage and rising velocity have been determined by the following relations. ${ }^{.1}$

$$
\begin{gathered}
\varepsilon_{b}=k\left(U-U_{m f}\right) / \bar{U}_{b} \\
\bar{U}_{b}=\left(U-U_{m f}\right)+0.711\left(g D_{b}\right)^{1 / 2}
\end{gathered}
$$

where $\bar{U}_{b}$ is related as half of $U_{b}$ in Fig. 1(b).

The variation of bubble size along the height as a function of an excess gas velocity $\left(U-U_{m f}\right)$ can be obtained by combining Eqs. (8), (9) and (10).

$$
d D_{b} / d h=2.264 \quad \varepsilon_{b}=2.264 k\left(U-U_{m f}\right) / \bar{U}_{b}
$$

subject to the initial condition, $D_{b}=D_{b 0}$ at $h=0$. Then the solution of Eq. (11) can be expressed as

$$
\begin{gathered}
\left(U-U_{m f}\right)\left(D_{b}-D_{b 0}\right)+0.474 g^{1 / 2}\left(D_{b}^{3 / 2}-D_{b 0}^{3 / 2}\right) \\
=2.264 k\left(U-U_{m f}\right) h
\end{gathered}
$$

Based on previous findings, ${ }^{1,2,5,11,17,20)}$ the mean value of $k$ was found to be 0.5 . Therefore, bubble size can be determined from Eq. (11) with $k$ value of 0.5 at the given gas velocity as a function of bed height and type of distributor within the freely bubbling state in fluidized beds. Combining bubble flow rate and $\mathrm{Eq}$. (12) gives $N_{f}$ values along the height above the distributor. by

The point bubble frequency has been determined

$$
N_{p}=\pi N_{f} D_{b f}^{2} / 4 A
$$

In the present study, the ratio of frontal to equivalent spherical bubble diameters used was 1.10 accounting for the mean wake fraction of $0.25 .{ }^{13)}$ The eruption to frontal bubble diameters used was $1.5^{2,5}$

\section{Experimental}

Experiments were carried out in a relatively large square column of $30 \times 30 \mathrm{~cm}$ cross section and $4.0 \mathrm{~m}$ height, as shown in Fig. 2. The main section of the column was constructed by flanging together two pieces of $30 \times 30 \mathrm{~cm} \times 2 \mathrm{~m}$ high plexiglas plate. The solid particles were supported on a perforated plate which contained 314 evenly spaced holes of $2 \mathrm{~mm}$ diameter and served as the gas distributor. The air flow rate was measured with a flow cell (Nippon Flow Cell Co.) and regulated by means of globe valves on the feed and bypass lines. Nine pressure taps were 


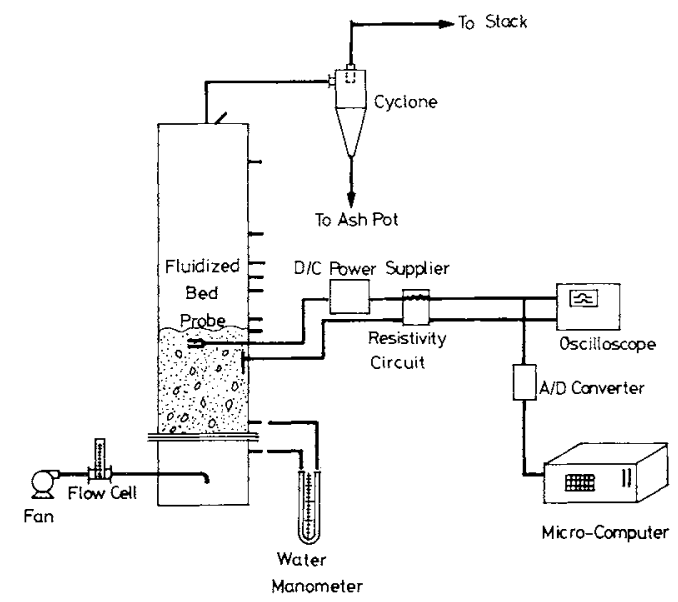

Fig. 2. Experimental apparatus

mounted on the wall of the column.

The solid particles have surface-mean diameters of 340,490 , or $620 \mu \mathrm{m}$. They were pulverized anthracite coal with apparent and bulk densities of 2.3 and $1.0 \mathrm{~g} /$ $\mathrm{cm}^{3}$, respectively.

To measure the bubble properties, an electroresistivity probe was constructed by using a $\mathrm{K}$-type thermocouple with a diameter of $0.85 \mathrm{~mm}$ (Fig. 2). The clearance between the two tips was $0.95 \mathrm{~cm}$.

Air with the desired superficial velocity was introduced into the bed formed by a known weight of solids. When steady state had been reached, the pressure profile along the entire height of the column was measured using water manometers. At the same time bubble properties were measured by using the resistivity probe at four bed heights $(10,22,30$ and $40 \mathrm{~cm}$ ) above the distributor and at six radial positions $(0,2.4,5.0,7.5,10.0$ and $12.5 \mathrm{~cm})$. About 600 bubbles were analysed at each experimental condition. The output signals from the bubble probe were stored in a microcomputer (Cromemco CS3HD5E) via an oscilloscope and an A/D converter. The bubble length and frequency were determined from the stored signals. The average number of signals per unit time at both tips was assumed to represent bubble frequency.

A typical signal is shown in Fig. 3. The average bubble rising velocity was calculated from the relation

$$
\bar{U}_{b, \text { mea }}=\frac{2}{N} \sum_{i=1}^{N}\left(\frac{S}{t_{a}+\bar{t}_{d}}\right)_{i}
$$

where $N, S, t_{a}$ and $t_{d}$ are total pairs of the lower and upper signals, clearance between the probe tips and time delay at both ends of a signal, respectively.

The average bubble length was obtained from

$$
l=\frac{1}{N} \sum_{i}^{N}\left[S\left(\frac{B_{l}+B_{u}}{t_{a}+t_{d}}\right)\right]
$$

where $B_{l}$ and $B_{u}$ are the times for a bubble to occupy

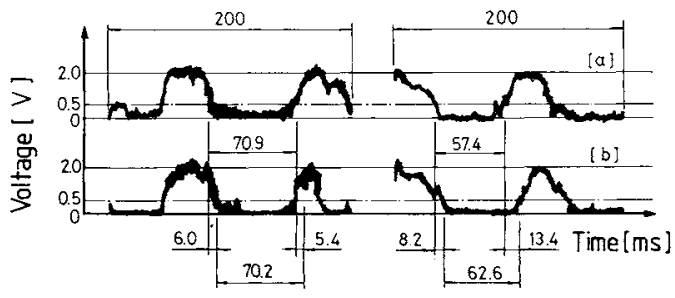

Fig. 3. Typical output signals from probe: (a), lower tip; (b), upper tip

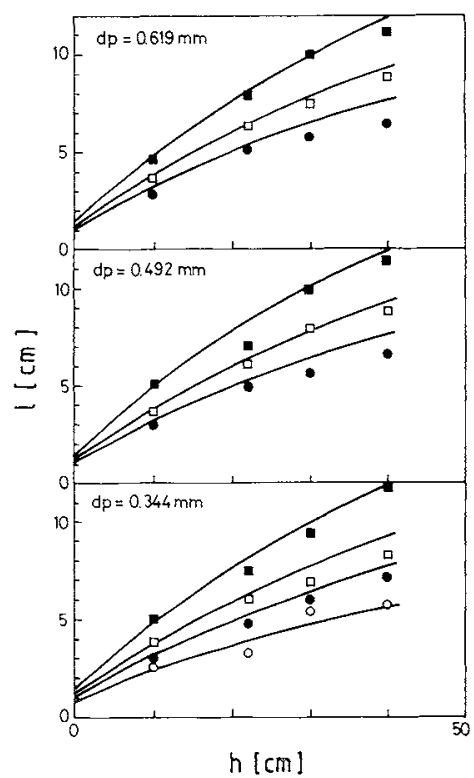

Fig. 4. Bubble length along bed height. $\left(U-U_{m f}\right)$ : $(\bigcirc) 4.7$; (•) $7.8 ;(\square) 10.9 ;(\mathbf{\square}) 17.1 ;(\longrightarrow)$ model

the lower and upper tips.

\section{Results and Discussion}

The variation of mean pierced bubble length along the height with gas velocity and particle size is shown in Fig. 4. As can be seen, the pierced bubble length increased with increase in bed height and gas velocity. In the figure, the solid lines are values calculated from Eq. (12), which predicts somewhat higher values than the experimental values since the probe measurement may give rather elongated values. However, the mean pierced bubble length was unaffected by particle size at the same excess gas velocity and height from the distributor.

The point bubble frequencies along the height as a function of gas velocity is shown in Fig. 5. The bubble frequency increased with gas velocity due to the increase in bubble flow rate. It decreased with bed height because of bubble coalescence. In the figure, solid lines represent the values calculated from Eq. (13). As can be seen, the calculated values accord well with the experimental values. In general, particle size did not affect the point bubble frequency at the same excess gas velocity and height from the distributor in 


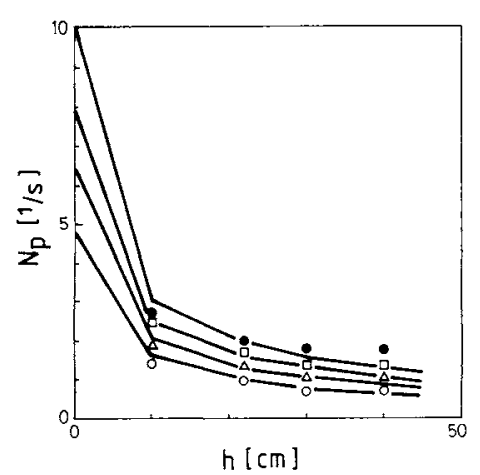

Fig. 5. Point bubble frequency along bed height $\left(d_{p}=\right.$ $0.344 \mathrm{~mm}$ ). $U(\mathrm{~cm} / \mathrm{s}):(\bigcirc) 12.4 ;(\triangle) 15.5$; ( $) 18.6$; (○) 24.8 ; $(\longrightarrow)$ model

this study.

The point bubble frequency and pierced bubble length in the radial direction are shown in Figs. 6(a) and (b), resepctively. As can be seen, the point bubble frequency and the bubble length are nearly constant in the radial direction except near the column wall at lower bed height. Therefore, assumption (3) of the present study may be a reasonable one.

The bubble rising velocity along the bed height as a function of excess gas velocity is shown in Fig. 7. As expected, the bubble rising velocity increased with excess gas velocity and bed height due to the increase in bubble size from the coalescence. The solid lines represent the bubble velocities from Eq. (10) with the bubble size from Eq. (12). As can be seen, Eq. (10) can predict well the present experimental data.

Good agreement can also be seen in Fig. 8(a) between the bubble size measurement of Hatate et $a l .^{6)}$ and the values from the present model equation. In general, the values of the present model lie between the values predicted from the correlation of Darton $e t$ $a l^{3)}$ and that of Mori and Wen. ${ }^{7)}$

The eruption bubble diameter along the bed height in the $22.9 \mathrm{~cm}$-ID fluidized bed of Fryer and Potter ${ }^{4}$ is shown in Fig. 8(b) as a function of gas velocity in which solid lines represent the model Eq. (12). This agreement is far better than that in the correlation of Darton et al. ${ }^{3)}$

Also, the data of eruption bubble diameter in the $30.8 \mathrm{~cm}^{-I D}$ of Geldart ${ }^{5)}$ were well represented by Eq. (12) with the $k$ value of 0.5 , as shown in Fig. 9. In the model calculation, the expanded bed height was determined ${ }^{1)}$ and the bubble size was estimated at the given height.

The data of eruption bubble diameters in the $0.61 \times$ $0.61 \mathrm{~m}$ square and $1.22 \times 1.22 \mathrm{~m}$ square fluidized beds with multi-tuyeres of Whitehead and Young ${ }^{17)}$ are compared with the present model equation in Fig. 10. It has been assumed that the number of tuyeres was the number of orifices of the distributor plate in the estimation of the initial bubble size. The bubble size

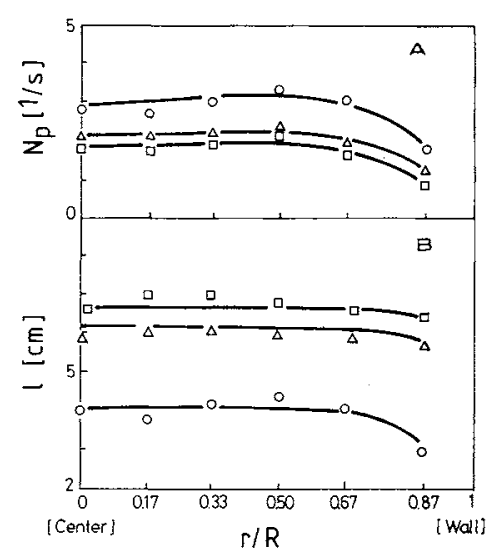

Fig. 6. Radial distribution of point bubble frequency and bubble length $\left(U=18.6 \mathrm{~cm} / \mathrm{s}, d_{p}=0.344 \mathrm{~mm}\right) . h(\mathrm{~cm})$ : (口) 30 ; $(\triangle) 22 ;(0) 10$

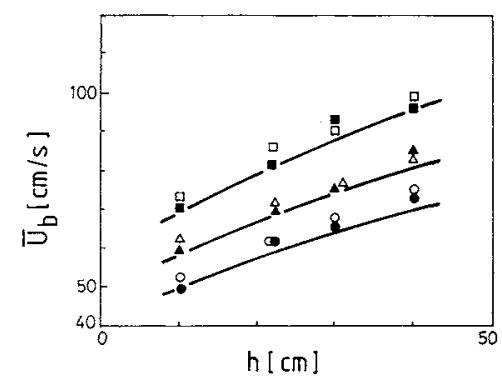

Fig. 7. Bubble rising velocity along bed height. $d_{p}(\mathrm{~mm})$, 0.492;U-U $U_{m f}(\mathrm{~cm} / \mathrm{s}):(\bigcirc) 7.8 ;(\triangle) 10.9 ;(\square) 17.1 . d_{p}(\mathrm{~mm})$, $0.619 ; U-U_{m f}(\mathrm{~cm} / \mathrm{s}):(\bullet) 7.8 ;(\boldsymbol{\Delta}) 10.9 ;(\boldsymbol{\square}) 17.1$
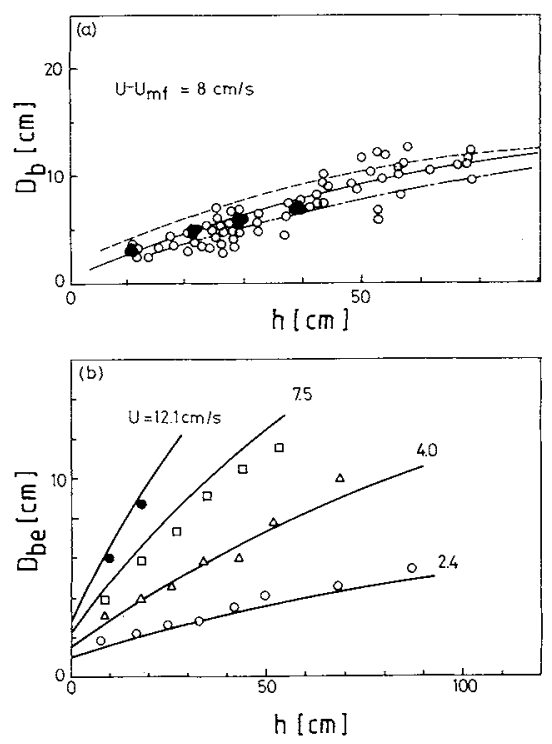

Fig. 8. Comparison between calculated bubble size along bed height and measured bubble size of [a]: $(O)$, Hatate $e t$ al. ${ }^{6)}\left[d_{p}=0.521 \mathrm{~mm}\right] ;(\bigcirc)$, this study $\left[d_{p}=0.344,0.492\right.$, $0.619 \mathrm{~mm}$; [b]: Fryer and Potter ${ }^{4)}\left[d_{p}=0.117 \mathrm{~mm}\right] ;(\longrightarrow)$ present model; (--_) Mori and Wen; ${ }^{7)}(-.-)$ Darton et $a l^{3)}$

was determined at the expanded bed height. As can be seen in the figure, the predicted bubble size agrees well with the measured values over the entire experimental 


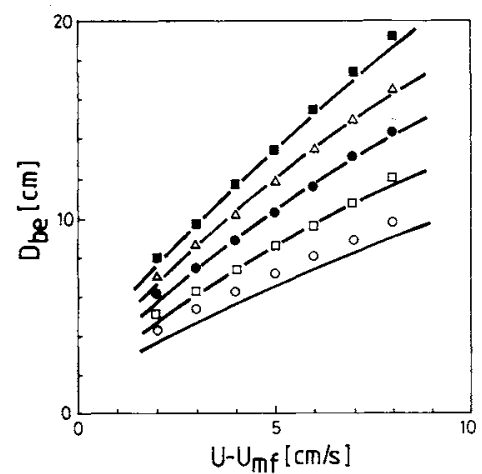

Fig. 9. Comparison between measured bubble size as a function of excess gas velocity of Geldart. ${ }^{5}\left[d_{p}=0.128 \mathrm{~mm}\right]$ and present model. $h_{m j}(\mathrm{~cm}):(\bigcirc) 20 ;(\square) 30 ;(\bullet) 40 ;(\triangle) 50$; (Ш) 60

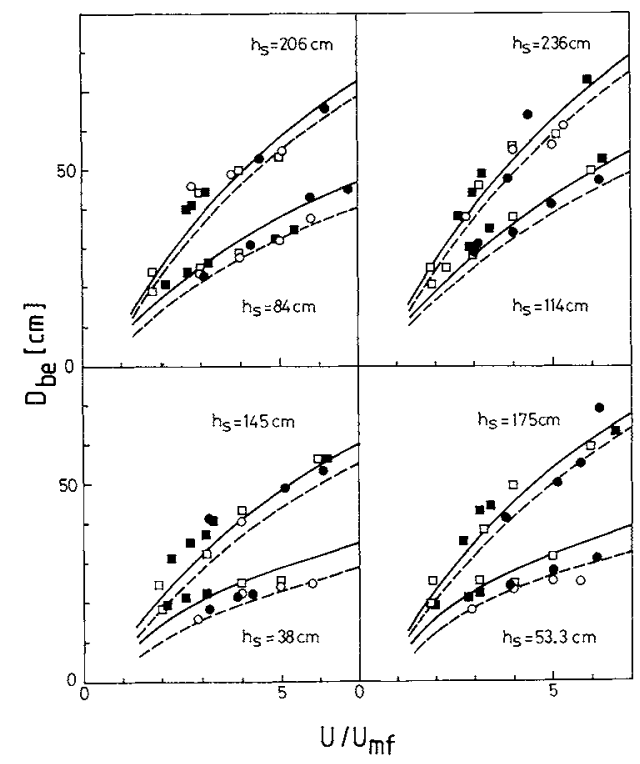

Fig. 10. Comparison between eruption bubble diameter of Whitehead and Young ${ }^{17)}\left[d_{p}=0.166 \mathrm{~mm}\right]$ as a function of $U /$ $U_{m f}$ and calculated values. $15.2 \mathrm{~cm}$ tuyere spacing: $(O)$ bed area of $61 \times 61 \mathrm{~cm}$; (O) bed area of $122 \times 122 \mathrm{~cm} ;(---)$ present model.

$30.5 \mathrm{~cm}$ tuyere spacing: ( $\square$ ) bed area of $61 \times 61 \mathrm{~cm}$; $(\boldsymbol{C})$ bed area of $122 \times 122 \mathrm{~cm}$; ( - ) present model

range. The present agreement is better than the predicted values of Rowe ${ }^{91}$ and Darton et al. ${ }^{31}$

The effect of excess gas velocity $\left(U-U_{m f}\right)$ on bubble diameter reported by Zhang et al. ${ }^{20)}$ is shown in Fig. 11(a). Their data were obtained in a bed of $0.92-1.2 \mathrm{~mm}$ particles at room temperature and in a coal combustor. As can be expected, bubble size increased with excess gas velocity. Bubble sizes from the correlations of the present and previous studies $^{2,3,7)}$ were compared with the experimental data of Zhang et al $^{20)}$ as shown in Fig. 11(a). The correlation values from Mori and $\mathrm{Wen}^{7)}$ and Darton et al. ${ }^{3)}$ predict rather smaller bubble diameters than the measured values except at lower excess gas velocity. However, the values from the correlation of Cranfield
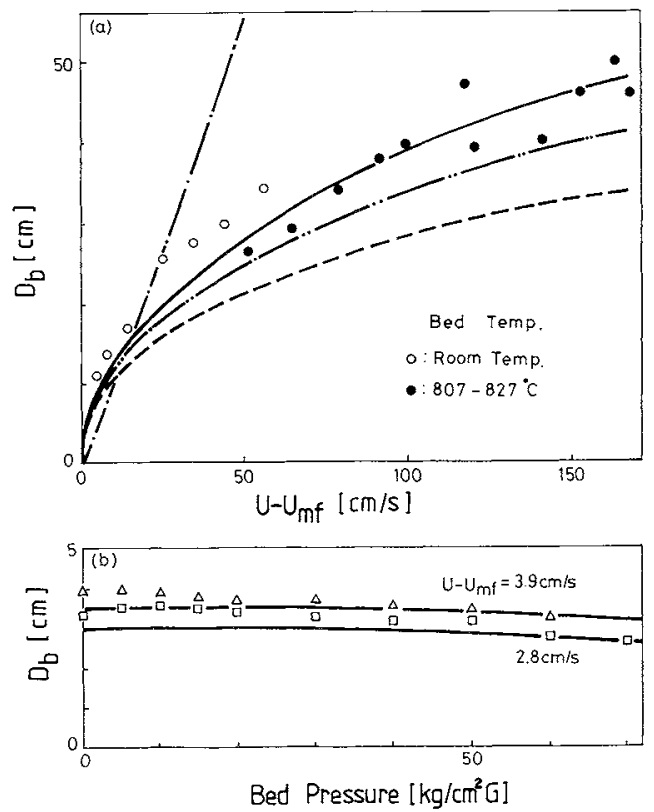

Fig. 11. Comparison between measured bubble size of Zhang et al. ${ }^{20)}\left[(a): d_{p}=0.922-1.2 \mathrm{~mm}\right]$ and Rowe et al.; ${ }^{11\}}$ [(b): $\left.d_{p}=0.45 \mathrm{~mm}\right]$ as a function of excess gas velocity and bed pressure and calculated values of model equations. ( - ) present model; (---) Darton et al., ${ }^{3)}$ (-..-) Mori and Wen; ${ }^{7}(-\cdot-)$ Cranfield and Geldart ${ }^{2)}$

and Geldart ${ }^{21}$ predict steeper increase in bubble size with excess gas velocity. As can be seen in the figure, the present correlation represents the bubble diameter well over a wide range of excess gas velocity. Therefore, it may be claimed that the present correlation can be utilized in the higher gas velocity ranges.

The effect of bed pressure on bubble size has been reported by Rowe et al. ${ }^{11)}$ The variation of bubble size with bed pressure as a function of excess gas velocity is shown in Fig. 11(b), in which the solid line represents the model calculation. In their experiments, it was observed that the solid particles behave as a group B powder in the Geldart classification at atmospheric pressure but as a group A powder at pressures above 2 bar. As can be seen, bubble size decreased slowly with bed pressure due to the increase in minimum bubbling velocity. Also, the present model equation fits the measured values well.

In addition, the present correlation values were compared with the measured values from the previous studies. ${ }^{2,4-6,10,11,14,15,17,19,20)}$ The measured bubble sizes were well represented by the present model equation within a standard deviation of $1.7 \mathrm{~cm}$.

Therefore, it may be claimed that the present correlation covers experimental variables in the particle size range of $0.041-1.760 \mathrm{~mm}$, bed diameters of $10-122 \mathrm{~cm}$, fluidizing gas velocities of $0.91-214 \mathrm{~cm} / \mathrm{s}$, bed temperatures of $20-1000^{\circ} \mathrm{C}$ and bed pressures of 1-71 bar. 


\section{Comparison of Correlations}

Rearranging Eq. (12) in a dimensionless form, we have

$$
\begin{aligned}
\frac{\left(U-U_{m f}\right)}{\sqrt{g D_{b}}} & =\frac{0.474\left\{1-\left(D_{b 0} / D_{b}\right)^{3 / 2}\right\} D_{b}}{2.264 k h-D_{b}+D_{b 0}} \\
& =0.474 \frac{\left(\sqrt{P_{0}}-1\right)}{\sqrt{P_{0}}} \frac{D_{b}}{2.264 k h-D_{b}+D_{b 0}}
\end{aligned}
$$

where $P_{0}$ is the number of initial bubbles gathered to grow to a bubble size $D_{b}$. In Eq. (16), the left-hand side indicates the Froude number based on the bubble movement. Thus, the equation represents approximately a linear relationship between the square root of the bubble's kinetic to its static energies and the average fractional change of square root of static energy during bubble rise in $D_{b}$ height. The previous correlations of Rowe $^{9)}$ and Darton et al. ${ }^{3)}$ are of similar form to Eq. (16). However, they have a different physical meaning as explained below.

The Froude number in Eq. (16) has a maximum value at the distributor $(h=0)$ for a given condition at which the following relation can be obtained.

$$
\frac{U-U_{m f}}{\sqrt{g D_{b 0}}}=\frac{0.711 \varepsilon_{b 0}}{k-\varepsilon_{b 0}}
$$

As can be seen, the Froude number increases with bubble voidage. This relation holds below the bubble voidage of $k$. It indicates that the $k$ value is the maximum bubble voidage in the bubbling fluidized bed. Also, it may be inferred that the bubble flow fraction of excess gas velocity is equal to the maximum initial bubble voidage at the distributor plate.

The bubble voidage increases with increase in fluidizing velocity. The increase of fluidizing velocity in Eq. (10) reduces the relative magnitude of $0.711 \sqrt{g D_{b}}$ compared to $\left(U-U_{m f}\right)$. In a relatively large fluidized bed without wall effect, the increase in fluidizing velocity may approach a condition of $\left(U-U_{m f}\right)>0.711 \sqrt{g D_{b}}$ and the bubble voidage becomes $k$ value in Eq. (9). If the bubbles are in a cubic array just touching each other, $\varepsilon_{b 0}$ and $k$ can be represented by $\pi / 6 .{ }^{16)}$ This value is very similar to the $k$ value of 0.5 which has been used in the present study.

Most of the reported correlations on bubble size have been measured in the lower ranges of excess gas velocity. Under this condition, if the height of measurement is far apart from the distributor, the $\left(\sqrt{P_{0}}-1\right) / \sqrt{P_{0}}$ term approaches unity. Then, Eq. (12) with $k$ value of 0.5 can be simplified with moderate error as

$$
D_{b}=\frac{1.787\left(U-U_{m f}\right)^{2 / 3}\left(h+D_{b 0} / 1.132\right)^{2 / 3}}{g^{1 / 3}}
$$

The above Eq. (18) is of similar form to the correlations of Rowe ${ }^{9)}$ and of Darton et al. ${ }^{3)}$ The dependency of bubble size on excess gas velocity is somewhat higher but is somewhat lower on the bed height. Under the lower excess gas velocity range, Eq. (18) can be rearranged as

$$
D_{b}=\frac{1.787\left(U-U_{m f}\right)^{2 / 3} h^{2 / 3}}{g^{1 / 3}}
$$

which is similar to the correlation of Yacono. ${ }^{18)}$

Near the distributor plate $\left(D_{b 0} / D_{b} \cong 1.0\right)$, Eq. (12) with $k$ value of 0.5 can be rearranged as

$$
\frac{U-U_{m f}}{\sqrt{g D_{b}}}=\frac{0.474\left\{1-\left(D_{b 0} / D_{b}\right)^{3 / 2}\right\}}{1.132 h / D_{b}}
$$

Then the bubble size can be represented by the following relation:

$D_{b}=\frac{1.787\left(U-U_{m f}\right)^{2 / 3}\left\{h+0.419 g^{1 / 2} D_{b 0}^{3 / 2} /\left(U-U_{m f}\right)\right\}^{2 / 3}}{g^{1 / 3}}$

This Eq. (21) is also similar to the correlations of Rowe $^{\text {9) }}$ and Darton et al. ${ }^{3)}$ However, it is somewhat different from Eq. (18). As can be seen from the above Eqs. (18)-(21), the published correlations ${ }^{3,9,18)}$ of bubble size are the specific conditions of the present correlation.

\section{Conclusions}

In the freely bubbling gas-fluidized bed, mean bubble growth can be interpreted by bubble coalescing behavior through the collision theory. The gradient of bubble size along the bed height has been correlated linearly with bubble voidage, assuming random spatial distribution of bubbles. The froude number based on the bubble movement can be represented approximately by a linear relationship with the average fractional change of square root of bubble static energy during their rise in $D_{b}$ height. The axial gradient of bubble size and Froude number increased with bubble voidage.

Experimental data on bubble size in the present and previous studies are well represented by the present model equation.

$$
\begin{aligned}
& \text { Nomenclature } \\
& A \quad=\text { bed area } \quad\left[\mathrm{cm}^{2}\right] \\
& B \quad=\text { time for a bubble occupying the probe } \quad[\mathrm{s}] \\
& D_{b} \quad=\text { equivalent spherical bubble diameter having } \\
& \text { same volume as that of bubble } \quad[\mathrm{cm}] \\
& D_{b e} \quad=\text { eruption bubble diameter } \quad[\mathrm{cm}] \\
& \begin{array}{lll}
D_{b f} & =\text { frontal bubble diameter } & {[\mathrm{cm}]}
\end{array} \\
& D_{b 0} \quad=\text { initial bubble diameter at distributor } \quad[\mathrm{cm}] \\
& \begin{array}{lll}
d_{p} & =\text { particle diameter } & {[\mathrm{cm}]}
\end{array}
\end{aligned}
$$




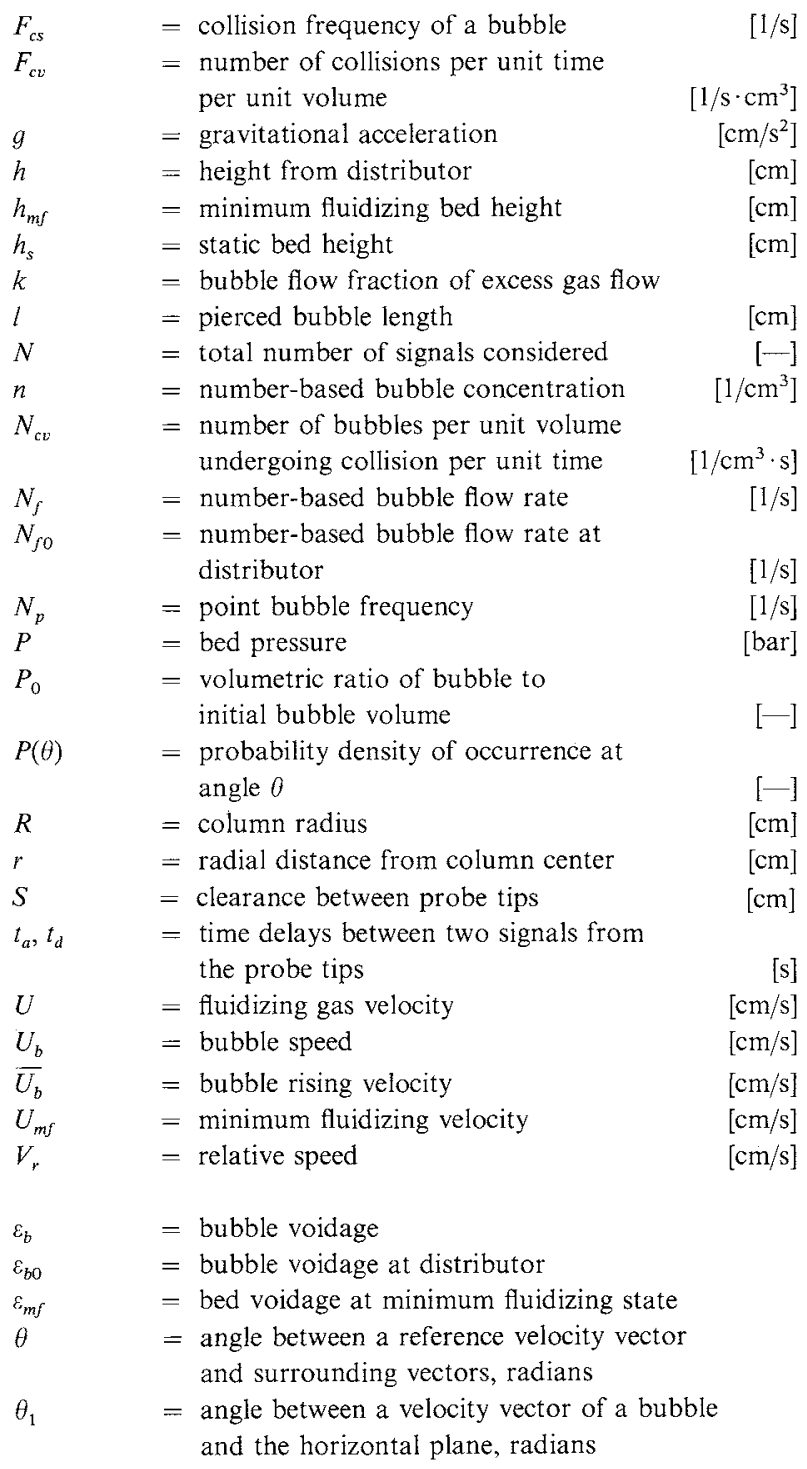

〈Subscripts〉

cal $\quad=$ calculated

$$
\begin{array}{ll}
l & =\text { lower } \\
\text { mea } & =\text { measured } \\
u & =\text { upper }
\end{array}
$$

cm] Literature Cited

1) Clift, R. and J. R. Grace: "Continuous Bubbling and Slugging," Fluidization, 2nd ed., Davidson and Harrison Eds., Academic Press, London (1985).

2) Cranfield, R. R. and D. Geldart: Chem. Eng. Sci., 29, 935 (1974).

3) Darton, R. C., R. D. La Nauze, J. F. Davidson and D. Harrison: Trans. Instn. Chem. Engr., 55, 274 (1977).

[1/s] 4) Fryer, C. and O. E. Potter: AIChE J., 22, 38 (1976).

5) Geldart, D.: Powder Technol., 4, 41 (1970/1971).

6) Hatate, Y., D. F. King, M. Migita and A. Ikari: J. Chem. Eng. Japan, 18, 99 (1985).

7) Mori, S. and C. Y. Wen: AIChE J., 21109 (1975).

8) Present, R. D.: "Kinetic Theory of Gases," McGraw-Hill, New York (1958).

9) Rowe, P. N.: Chem. Eng. Sci., 31, 285 (1976).

10) Rowe, P. N. and D. F. Everett: Trans. Instn. Chem. Engr., 50, 55 (1972).

11) Rowe, P. N., P. U. Foscolo, A. C. Hoffman and J. G. Yates: Proc. 4th Int. Conf. on Fluidization, p. 53 (1983).

12) Rowe, P. N. and J. A. Goldsmith: 2nd Iranian Congr. Chem. Eng., Teheran (1975).

13) Rowe, P. N. and B. A. Partridge: Trans. Instn. Chem. Engr., 43, T157 (1965).

14) Stubington, J. F., D. Barrett and G. Lowry: Chem. Eng. Res. Des., 62, 173 (1984).

15) Werther, J.: AIChE National Meeting, Detroit (1973).

16) Werther, J., D. Bellgardt, H. Groenewald and K. Hilligardt: Proc. 9th Int. Conf, on Fluidized Bed Combustion, Vol. 1, p. 515 (1987).

17) Whitehead, A. B. and A. D. Young: Proc. Int. Symp. on Fluidization, Netherlands Univ. Press, Amsterdam, Vol. 1, p. 515 (1967).

18) Yacono, C. X. R.: Ph. D. Dissertation, Univ. of London, London (1975).

19) Yasui, G. and L. N. Johanson: AIChE J., 4, 445 (1958).

20) Zhang, M. C., P. M. Walsh and J. M. Beer: Proc. 7th Int. Conf. on Fluidized Bed Combusion, Vol. 1, p. 77 (1982). 